http://sciforum.net/conference/ece-1

Conference Proceedings Paper - Energies „, Whither Energy Conversion? Present Trends, Current Problems and Realistic Future Solutions"

\title{
Energy Efficiency through an On-Line Learning Approach for Forecasting of Indoor Temperature
}

\section{F. Zamora-Martínez, P. Romeu, P. Botella-Rocamora, J. Pardo}

1 Embedded Systems and Artificial Intelligence Group (ESAI). Escuela Superior de Enseñanzas Técnicas. Universidad CEU Cardenal Herrera. C/. San Bartolomé, n55. 46115, Alfara del Patriarca. Valencia. Spain ; E-Mail: \{francisco.zamora, pablo.romeu, pbotella, juan.pardo\}@uch.ceu.es

* Author to whom correspondence should be addressed; E-Mail: juan.pardo@uch.ceu.es; Tel.: +34-136-90-00; Fax: +34-130-09-77.

Received: 15 January 2014 / Accepted: 13 March2014 / Published: 14 March 2014

\begin{abstract}
University CEU Cardenal Herrera (CEU-UCH) has constructed a Solar house, known as SMLSystem, to participate in the Solar Decathlon Europe competition. A lot of technologies were integrated to reduce its overall power consumption. Among them, a predictive system, based on Artificial Neural Networks (ANNs), was developed. Such system produces short-term forecast of indoor temperature, using as input the data captured by a complex monitoring system. The system expects to reduce the power consumption mainly related to Heating, Ventilation and Air Conditioning (HVAC).

This paper presents preliminary results about viability of the development of such kind of predictive systems but for totally unknown environments, that is, without historical data. To do that it is possible to apply on-line learning approaches, where the model parameters are estimated following Gradient Descent (GD) methods, starting from a totally random model. Preliminary experimental results show a high forecasting accuracy with simple models and with a short training time of 4-5 days.
\end{abstract}

Keywords: Energy efficiency; Time series forecasting; Gradient descent; Artificial neural networks 


\section{Introduction}

The School of Technical Sciences at the University CEU-UCH has constructed a solar-powered house, known as Small Medium Large System (SMLsystem), to participate in the 2012 Solar Decathlon Europe competition [1]. It is a house, which integrates a whole range of different technologies to improve energy efficiency, allowing it to be a near-zero consuming energy house. The objective of the competition is about energy efficiency. The reason is that nowadays, in European Union, primary energy consumption in buildings represents about $40 \%$ of the total [2], where more than a half is used by HVAC (Heating, Ventilating and Air Conditioning) systems [3]. The activation/deactivation of such systems depends on comfort parameters, being one of the most important the indoor temperature, directly related with the notion of comfort. Hence there is a need to design and implement new systems at home, which should be able to produce and use energy efficiently and wisely, reaching a balance between consumption and streamlined comfort.

To implement such intelligent systems, forecasting techniques of the area of artificial intelligence can be applied. In fact, machine-learning techniques, as Artificial Neural Networks (ANNs), have been widely used for a range of applications in the area of energy systems modeling [4]. Of special interest to this area is the use of ANNs for forecasting room air temperature as a function of forecasted weather parameters (mainly solar radiation and air temperature) and actuator (heating, ventilating, cooling) state or manipulated variables, and the subsequent use of these mid/long-range prediction models for a more efficient temperature control, both in terms of regulation and energy consumption. The present work is focused on multivariate forecasting using as input features different weather indicators, based on a previous work [4]. Forecasting could be useful to reduce energy consumption of the HVAC system, but it is a complex task because of the multiple and different scenarios where a house could be constructed.

\section{Forecasting of time series}

Forecasting techniques are useful in terms of energy efficiency because they can help to develop predictive control systems. Time series, as the indoor temperature, are data series with trend and pattern repetition through time. Time series forecasting could be grouped as univariate and multivariate. But multivariate approaches could perform better than univariate when additional variables cause variations on the predicted target, as our case study. Such forecasting models are estimated given different parameters: number of past values, size of the future window, and position in the future of the prediction (future horizon). Depending on the size of future window, and how it is produced, forecasting approaches are denoted as: single-step-ahead forecasting, if the model forecasts only the next time step; and multi-step-ahead iterative forecasting, if the model forecasts only the next time step, but producing longer windows by an iterative process. Following this last approach, two different major kinds of model exist: Pure direct, that uses $\mathrm{Z}$ forecasting models, one for each possible future horizon; and Multiple Input Multiple Output (MIMO), that uses one model to compute the full $\mathrm{Z}$ future window. The last one has several advantages due to the joint learning of inputs and outputs that allows the model to learn the stochastic dependency between predicted values. Finally, the performance of forecasting methods over one time series could be assessed by several different 
evaluation functions which measure the empirical error of the model. For a deep analysis of the results, it has been used two different error functions: Mean Absolute Error (MAE) and Root Mean Square Error (RMSE).

\subsection{Forecasting methods}

Gradient Descent (GD) approach has been used to optimize the model parameters following the direction of the gradient, that minimizes a given loss function. The learning is performed following an on-line paradigm. Traditionally, the different learning modes are [5]:

- Batch mode: this scheme allows using accurate and fast matrix operations, but is not feasible with large datasets.

- On-line mode: this mode has faster convergence than batch, but could be noisier.

- Mini-batch mode: a trade-off between both above strategies. It has several computational advantages compared with on-line mode, allowing accurate and fast matrix operations.

However, in some real scenarios an on-line training mode will work better to estimate patterns at real time from the given data source. It allows to place untrained systems in their target location, expecting to achieve better model predictions as the time (running and learning) goes on. The idea is to find an alternative solution to the learning processes based on large training datasets, as traditionally occurs.

\section{Results and Discussion}

In our experimental database, time series were measured with a sampling period of $\mathrm{T}=1$ minute. However, in order to compute forecasting over less noisy signals, each time series was subsampled with a period of $T^{\prime}=15$ minutes, computing the mean of last $T^{\prime}$ values. One time feature and five sensor signals were taken into consideration and multiple covariates combinations plus different ANN topologies were also tested, it can be found at a previous work [4]. But the following three were found to be the most relevant for the present work: indoor temperature, hour feature and sun irradiance.

Extending previous experimentation, this paper analyses the viability of an on-line learning approach for indoor temperature forecasting. Results of a simple linear model (perceptron) are shown at Figure 1, learned following GD algorithm known as Back-propagation. Good MAE and RMSE values has been achieved with short training time, showing a trend to improve the error while the training continues. However, more experiments are necessary for a deeper future research.
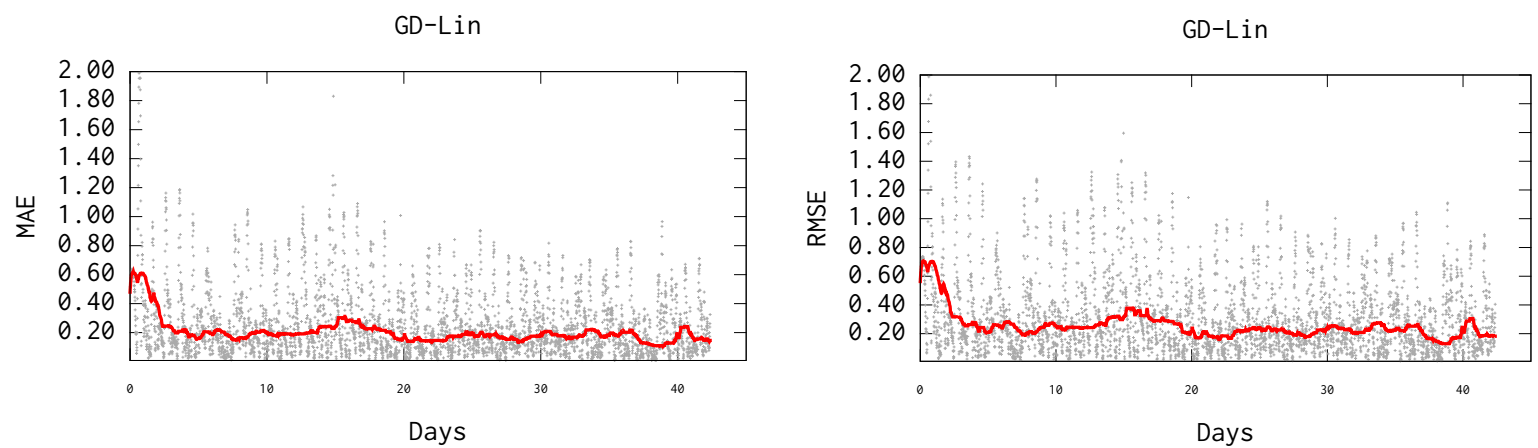

Figure 1 MAE and RMSE plot of every time instant (dots), and a median trend value computed with a sliding window of two days width. 


\section{Conclusions}

An on-line learning approach was slightly presented, which allows the integration of predictive models in totally unknown scenarios. Such approach has been tested in a real Solar house that was constructed for Solar Decathlon Europe competition. The model has been trained continuously, expecting to achieve better performance during time. A Gradient Descent (GD) on-line algorithm has been studied, using linear models and showing promising performance results. More complex models will be trained for comparative purposes in future work extensions. The model shows a good convergence behavior, but a deeper analysis is needed in order to state the dependence between the dataset size and the model complexity.

\section{Acknowledgments}

This work has been supported by Banco Santander and University CEU Cardenal Herrera through the project Santander-PRCEU-UCH07/12.

\section{Conflicts of Interest}

"The authors declare no conflict of interest".

\section{References and Notes}

1. United States Department of Energy, Solar Decathlon Europe Competition, 2012. http://www.solardecathlon.gov.

2. P. Ferreira, A. Ruano, S. Silva, E. Conceicao, Neural networks based predictive control for thermal comfort and energy savings in public buildings, Energy and Buildings 55 (2012) 238251. Cool Roofs, Cool Pavements, Cool Cities, and Cool World.

3. J. Alvarez, J. Redondo, E. Camponogara, J. Normey-Rico, M. Berenguel, P. Ortigosa, Optimizing building comfort temperature regulation via model predictive control, Energy and Buildings (2012).

4. F. Zamora-Martínez, P. Romeu, P. Botella-Rocamora, J. Pardo. Towards Energy Efficiency: Forecasting Indoor Temperature via Multivariate Analysis, Energies 6 (2013) 4639-4659.

5. R. O. Duda, P. E. Hart, D. G. Stork, Pattern Classification, second ed., John Wiley and Sons, 2001.6.

(C) 2014 by the authors; licensee MDPI, Basel, Switzerland. This article is an open access article distributed under the terms and conditions of the Creative Commons Attribution license (http://creativecommons.org/licenses/by/3.0/). 